\title{
A Repulsive Interaction in Classical Electrodynamics
}

\author{
Katalin Gambár ${ }^{1}$, Mario C. Rocca ${ }^{2}$, Ferenc Márkus ${ }^{3}$ \\ ${ }^{1}$ Institute of Microelectronics and Technology, Kálmán Kandó Faculty of \\ Electrical Engineering, Óbuda University, Tavaszmező u. 17, H-1084 Budapest, \\ Hungary \\ ${ }^{2}$ Departamento de Física, Faculdad de Ciencias Exactas, Universidad Nacional de \\ La Plata, C.C. 67, 1900 La Plata, Argentina \\ ${ }^{3}$ Deparment of Physics, Budapest University of Technology and Economics, \\ Budafoki út 8, H-1521 Budapest, Hungary \\ gambar.katalin@kvk.uni-obuda.hu,rocca@ fisica.unlp.edu.ar, \\ markus@phy.bme.hu
}

\begin{abstract}
Herein, we introduce an additional term into the induction equation (one of the Maxwell's equation). The related Lagrangian formalism applying the scalar and vector potentials is fitted to this modified Maxwell's equations. In the framework of Hamiltons's principle we are able to deduce Klein-Gordon equations with negative "mass term" for the field variables electric field $\boldsymbol{E}$ and magnetic induction $\boldsymbol{B}$. We can conclude from the mathematical structure of the equations that a repulsive interaction appears. The Wheeler propagator can be calculated for the present case by which the time evolution of the field can be discussed. In spite of the situation that these equations have tachyon solutions, the results are in line with the causality principle. As a consequence of the theory, a spontaneous charge disjunction process may rise in the field.
\end{abstract}

Keywords: Maxwell's equations; Klein-Gordon equation with negative "mass term"; Lagrangian, Wheeler propagator; charge distribution

\section{Introduction}

Mechanical [1, 2], thermodynamic [2-4] and further field theoretical examples [57] for the Klein-Gordon equation with negative "mass term" involve the same dynamical phase transition that operates between the diffusive and the wave type dynamics. Studying the existence of these kinds of phenomena we may assume that the occurrence of these are more general and not restricted exclusively to a certain part of physics. 
In the last decades a wider study of the negative mass term Klein-Gordon equation has been accomplished to get a detailed impression about the efficiency and the validity of this formulation [8-14]. This kind of examination is not a gratuitous mathematical whim at all, because there exist more realistic processes which are described by such equations. Thus, there is no reason to doubt their reality [15].

The mechanical example [1,2] is a stretched string lying on the diameter of a rotating disk when the appearing centrifugal force behaves as a repulsive interaction as Fig. 1 shows.

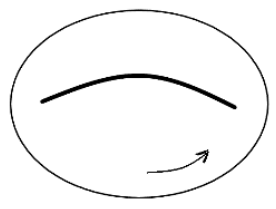

Fig. 1

Stretched string of a rotating disk

The model is covered by the equation:

$\frac{\partial^{2} \Psi}{\partial t^{2}}-\frac{F}{\rho A} \frac{\partial^{2} \Psi}{\partial x^{2}}-\omega_{0}^{2} \Psi=0$

where $\Psi$ is the displacement from the equilibrium position, $\rho$ is the mass density of the string, $F$ is the stretching force, $A$ is the cross section of the string and $\omega_{0}$ is the angular velocity of the disk. The second term pertains to the spring force, i.e., it is an attractive interaction. The so-called negative "mass term" is the third term of this equation due to the negative sign of the term. (This term is positive in the "well behaved" Klein-Gordon equation [16, 17]). The change of dynamics can be understood form the following physical picture. If the angular velocity $\omega_{0}$ is small enough, the spring vibrates around its equilibrium position, but above a certain threshold angular velocity the centrifugal force elongates the spring towards the bigger radius without vibration. This is a transition between the vibrating and the dissipative state, i.e., this a dynamic phase transition. The detailed studies can be done by the examination of dispersion relation $[1,2]$

$$
\omega\left(k, \omega_{0}\right)=\sqrt{\frac{F}{\rho A} k^{2}-\omega_{0}^{2}}
$$

Waves modes exist if

$$
\frac{F}{\rho A}>\frac{\omega_{0}^{2}}{k^{2}}
$$

and there are no wave modes if

$$
\frac{F}{\rho A}<\frac{\omega_{0}^{2}}{k^{2}}
$$


It is clear that the third term in Eq. (1) behaves as a repulsive interaction.

A much more interesting dynamic transition can be found in between wave like thermal propagation and the Fourier heat conduction. To achieve the aim, first, the equation of motion of Lorentz invariant thermal energy propagation [2-7] must be formulated:

$\frac{1}{c^{2}} \frac{\partial^{2} \mathrm{~T}}{\partial t^{2}}-\frac{\partial^{2} \mathrm{~T}}{\partial x^{2}}-\frac{c^{2} c_{v}^{2}}{4 \lambda^{2}} T=0$

It is obvious that the structure of this equation - aside from the meaning of the parameters - is the same as in Eq. (1). Here, $c$ is the speed of light; $c_{v}$ is the heat capacity and $\lambda$ is the heat conductivity, both of them are constant parameters, now. This equation implies the case of classical Fourier heat conduction [18]

$c_{v} \frac{\partial T}{\partial t}-\lambda \frac{\partial^{2} T}{\partial x^{2}}=0$

which solution is separated from the wave-like propagation by a dynamic phase transition via a spinodal instability $[19,20]$. This transition is also describable via the dispersion relation $[3,4]$

$$
\omega(k)=\sqrt{c^{2} k^{2}-\frac{c^{4} c_{v}^{2}}{4 \lambda^{2}}}
$$

The propagation is wave-like if:

$$
k>\frac{c c_{v}}{2 \lambda}
$$

and dissipative if:

$$
k>\frac{c c_{v}}{2 \lambda}
$$

Similarly, to the mechanical example, the third term introduces a certain repulsive interaction in the thermal energy propagation.

The present work is based on the idea of the previous examples. It seems interesting to formulate a Klein-Gordon type equation with the above mathematical structure with negative "mass term" - to electrodynamics problems and to examine what kind of physical process may be governed by this way. Now, the whole description remains within the framework of the classical electrodynamics [21, 22]. It will be shown that to achieve this aim we should add an extra term to one of the Maxwell's equations (see later Eq. (3b). The Lorentz invariance of the theory can be completed by the careful choice of this term. As the examples show, the meaning of the appeared term in the Klein-Gordon type equation should be a repulsive interaction. In the light of our knowledge by calculating the propagator of the process $[8,9]$ we point out that this repulsive interaction may cause a large spontaneous charge disjunction in the electric conductive medium. 
We mention that in several areas of modelling of natural processes brave steps are needed, since the phenomena include not negligible - but not obviously deducible - additional interactions [23-26].

The structure of the paper is as follows. In Chapter 2 the mathematical construction is elaborated that leads to a repulsive interaction in the electrodynamics. The method is based on this new idea to add an extra term to one of the Maxwell's equations. Finally, a negative mass term Klein-Gordon equation is obtained. As the propagators can generate from an initial state to a final state of a system in time, to achieve to time evolution of the electric and magnetic fields, and mainly the charge density, the so-called Wheeler propagator is needed calculate for this kind of KleinGordon equation. Applying the result of previous studies [8-14] this calculation is shown in Chapter 3. The evolution of electric field and the charge density is calculated analytically in Chapter 4, while a numerical calculation is presented for a simple data set for the charge densities at different time in Chapter 5. The conclusions and some final remarks are presented in Chapter 6.

\section{A Repulsive Force in the Electrodynamics}

Our present aim is to formulate those kind of equations of motion that preserve the Lorentz invariance of the theory providing the Klein-Gordon type equation with the negative "mass term". At this stage it is not seen immediately what kind of physical process is generated really, but we know that the physical meaning of this negative term is a repulsive interaction. We restrict our consideration to only a pulse-like impact within the system, thus the additional term in the Maxwell's equation should be a process starting at the initial $t_{0}$ and ending at the final $t$, i.e., assuming that the elapsed time $\tau$ of $\sim 10^{-18}$ to $10^{-12}$ seconds, is very short.

To achieve our previously summarized goals we start from the regular form of the Maxwell's equations [21, 22] modifying the second one (Eq. (3b) with an additional term

$\alpha^{2} \int_{0}^{t} \boldsymbol{B}\left(r, t^{\prime}\right) d t^{\prime}$

that brings a short time $(0<t<\tau)$ interaction in the theory. Thus the four equations take the form:

$$
\begin{aligned}
& \frac{1}{\mu_{0}} \operatorname{rot} \boldsymbol{B}=\varepsilon_{0} \frac{\partial \boldsymbol{E}}{\partial t}+\boldsymbol{J} \\
& \operatorname{rot} \boldsymbol{E}=-\frac{\partial \boldsymbol{B}}{\partial t}+\alpha^{2} \int_{0}^{t} \boldsymbol{B}\left(r, t^{\prime}\right) d t^{\prime} \\
& \varepsilon_{0} \operatorname{div} \boldsymbol{E}=\rho \\
& \operatorname{div} \boldsymbol{B}=0
\end{aligned}
$$


Here, $\boldsymbol{B}$ denotes the magnetic field, $\boldsymbol{E}$ is the electric field, $\boldsymbol{J}$ is the current density, $\rho$ is the charge density, $\varepsilon_{0}$ is the vacuum permittivity and $\mu_{0}$ is the vacuum permeability. The parameter $\alpha$ pertains to the assumed interaction. (The square is used for the later calculation convenience.)

Now, we show the calculations to understand the influence of the above extra term (Eq. (3)) in the theory. In order to solve these equations it is usual to introduce the vector potential $\boldsymbol{A}[21,22]$ by the help of Eq. (3d) as:

$\boldsymbol{B}=\operatorname{rot} \boldsymbol{A}$

Substituting this into Eq. (3b) and rearranging the obtained formula we get

$\operatorname{rot}\left(\boldsymbol{E}+\frac{\partial \boldsymbol{A}}{\partial t}-\alpha^{2} \int_{0}^{t} \boldsymbol{A}\left(r, t^{\prime}\right) d t^{\prime}\right)=0$

We can express the electric field $\boldsymbol{E}$ from the above equation

$\boldsymbol{E}=-\frac{\partial \boldsymbol{A}}{\partial t}-\operatorname{grad} \varphi+\alpha^{2} \int_{0}^{t} \boldsymbol{A}\left(r, t^{\prime}\right) d t^{\prime}$

where the scalar potential $\varphi$ is introduced too. Since there is a free degree of freedom in the connection of the scalar and the vector potentials, we are allowed to take the condition:

$\frac{\partial \varphi}{\partial t}+\operatorname{div} \boldsymbol{A}=0$

with the choice

$\varepsilon_{0} \mu_{0}=1$

for these universal parameters. Now, we take Eq. (3c) and we replace $\boldsymbol{E}$ into it, thus we can write

$\operatorname{div} \boldsymbol{E}=-\frac{\partial(\operatorname{div} \boldsymbol{A})}{\partial t}-\Delta \varphi+\alpha^{2} \int_{0}^{t} \operatorname{div} \boldsymbol{A}\left(r, t^{\prime}\right) d t^{\prime}=\frac{\rho}{\varepsilon_{0}}$

Eliminating the vector potential by the help of Eq. (7), thus we obtain

$\frac{\partial^{2} \varphi}{\partial t^{2}}-\Delta \varphi-\alpha^{2} \varphi=\frac{\rho}{\varepsilon_{0}}$

The third term is a Lorentz invariant negative mass term Klein-Gordon equation. We remember that the term $-\alpha^{2} \varphi$ acts just in the time interval $\tau$. The structure of equation is similar to the equations (1) and (2) (such as in Refs. [1-7]) that includes a dynamical phase transition depending on the parameter $\alpha$ as a consequence of a spinodal instability $[19,20]$. The equation for the vector potential can be also formulated, starting from Eq. (3a) and substituting the form of electric field from Eq. (6)

$\operatorname{rotrot} \boldsymbol{A}=-\frac{\partial^{2} \boldsymbol{A}}{\partial t^{2}}-\frac{\partial \operatorname{grad} \varphi}{\partial t}+\alpha^{2} \boldsymbol{A}+\mu_{0} \boldsymbol{J}$

Applying the vector identity: 
rotrot $=$ graddiv $-\Delta$

and the condition in Eq. (7), we can rewrite the above equation in a more expressive form

$$
\frac{\partial^{2} \boldsymbol{A}}{\partial t^{2}}-\Delta \boldsymbol{A}-\alpha^{2} \boldsymbol{A}=\mu_{0} \boldsymbol{J}
$$

which is also a Lorentz invariant expression, and we can recognize that this is also a negative mass term Klein-Gordon type equation for the vector potential $\boldsymbol{A}$. Similarly to the previous remark, the term $-\alpha^{2} \boldsymbol{A}$ is active in the time range $\tau$. We can conclude that both the scalar and the vector potentials as basic fields - the components of a four vector

$A_{\mu}=(-\varphi, \boldsymbol{A})$

- fulfill the Lorentz invariant Klein-Gordon type equations with the same mathematical structure, the field variables propagate with the same speed, the whole description is Lorentz invariant.

It is important to emphasize that - from the viewpoint of the physical process description - the Lorentz invariant field equations (equations 9 and 11) for the scalar and the vector potentials have central roles. All of the other field variables can be deduced from these potentials $[21,22,27,28]$. This fact can be obviously seen from the Lagrangian of the theory formulated $[29,30]$ as

$L=-\frac{1}{4} F_{\mu \nu} F^{\mu \nu}-\frac{1}{2} \lambda\left(\partial_{\mu} A^{\mu}\right)^{2}+\frac{1}{2} \alpha^{2} A_{\mu} A^{\mu}+j_{\mu} A^{\mu}$

where the $F_{\mu \nu}$ is the electromagnetic tensor field

$F_{\mu \nu}=\partial_{\mu} A_{\nu}-\partial_{\nu} A_{\mu}$

Selecting $\lambda=1$ (Feynman's gauge) [31,32] in the Lagrangian the movement equations for the four-potential can be obtained for the present case. It is clear that the electric and magnetic fields are not observables and are not components of neither the electromagnetic tensor field nor the Lagrangian.

Now, we should write the equations for the field variables, $\boldsymbol{E}$ and $\boldsymbol{B}$. Thus, we take the time derivative of Eq. (3a)

$\operatorname{rot} \frac{\partial \boldsymbol{B}}{\partial t}=\frac{\partial^{2} E}{\partial t^{2}}+\mu_{0} \frac{\partial \boldsymbol{J}}{\partial t}$

The term on the left hand side can be substituted after taking the rotation of Eq. (3b) by which we write

$$
\operatorname{rot}\left(-\operatorname{rot} \boldsymbol{E}+\alpha^{2} \int_{0}^{t} \boldsymbol{B}\left(\boldsymbol{r}, t^{\prime}\right) d t^{\prime}\right)=\frac{\partial^{2} \boldsymbol{E}}{\partial t^{2}}+\mu_{0} \frac{\partial \boldsymbol{J}}{\partial t}
$$

We can eliminate the field $\boldsymbol{B}$ applying again Eq. (3a), and finally we obtain

$$
\frac{\partial^{2} \boldsymbol{E}}{\partial t^{2}}-\Delta \boldsymbol{E}-\alpha^{2} \boldsymbol{E}=-\frac{1}{\varepsilon_{0}} \operatorname{grad} \rho-\mu_{0} \frac{\partial \boldsymbol{J}}{\partial t}+\mu_{0} \alpha^{2} \int_{0}^{t} \boldsymbol{J}\left(\boldsymbol{r}, t^{\prime}\right) d t^{\prime}
$$


Similarly, for the field $\boldsymbol{B}$, we take the time derivative of Eq. (3b)

$\operatorname{rot} \frac{\partial \boldsymbol{E}}{\partial t}=-\frac{\partial^{2} \boldsymbol{B}}{\partial t^{2}}+\alpha^{2} \boldsymbol{B}$

and eliminating the field $\boldsymbol{E}$ by the help of the rotation of Eq. (3a) we obtain the equation for the magnetic field

$\frac{\partial^{2} \boldsymbol{B}}{\partial t^{2}}-\Delta \boldsymbol{B}-\alpha^{2} \boldsymbol{B}=\mu_{0}$ rot $\boldsymbol{J}$

It can be seen that for all of the field equations - equations (9), (11), (16) and (18) - have the same structure. We know from the former studies [1-7, 15] that these Klein-Gordon equations with a negative "mass term" are resulted from repulsive interactions. Thus, it seems to us that the interaction in the present case is a repulsive-like force which appears mathematically in the second Maxwell's equation, in Eq. (3b).

\section{The Wheeler Propagator and the Time-Evolution of the Electric Field}

The following physical description of the Wheeler propagator is based on Feynman's and Wheeler's original idea [33,34]. The clear mathematical deduction of the Wheeler propagator is developed by Bollini, Rocca, Giambiagi and Oxman [8-14]. The difficult and complicated mathematical method to evaluate the calculations needs to apply the Bochner's theorem [35, 36] taking into account further complicated mathematical formulations [37].

In the knowledge of the time evolution equations we can study the processes evolving in the electric conductive media. We focus on the connection between the appearing electrical field and the charge distribution given by Eq. (16). Since the last two terms of this equation make rather complicated the solution and assuming that the contribution of the current and the time derivative of the current can be negligible at the initial time, we can simplify the problem to:

$\frac{\partial^{2} \mathbf{E}}{\partial t^{2}}-\Delta \boldsymbol{E}-\alpha^{2} \boldsymbol{E}=-\frac{1}{\varepsilon_{0}} \operatorname{grad} \rho$

This equation can be solved applying the Green function method. On the basis of it, the electric field $\boldsymbol{E}$ as the solution of this partial differential equation can be expressed by the following integral:

$\boldsymbol{E}(r, t)=\int-\frac{1}{\varepsilon_{0}} \operatorname{grad} \rho\left(x^{\prime}\right)\left[\frac{1}{(2 \pi)^{4}} \int d^{4} k \frac{e^{i k\left(x-x^{\prime}\right)}}{k^{2}-\alpha^{2}}\right] d V^{\prime}$

Here, the four-vector:

$x=\left(t=x_{0}, r=\left(x_{1}, x_{2}, x_{3}\right)\right)$ 
involves both the space and time coordinates:

$k=\left(\omega=p_{0}, k\right)$

denotes the four-momentum

$d V^{\prime}=d x_{1}^{\prime} d x_{2}^{\prime} d x_{3}^{\prime}$

is the volume element. (We follow the notations of Refs. [8-14] in the calculations of the Wheeler propagator.) The expression

$G\left(x, x^{\prime}\right)=\frac{1}{(2 \pi)^{4}} \int d^{4} k \frac{e^{i k\left(x-x^{\prime}\right)}}{k^{2}-\alpha^{2}}$

in the $[\ldots]$ bracket is the Green function generating the evolution of the process in the space-time from the initial $x^{\prime}$ to the final $x$.

In order to evaluate this integral, we find the zero points of the denominator

$k^{2}-\alpha^{2}=p^{2}-p_{0}^{2}-\alpha^{2}=0$

from which we obtain

$p_{0}= \pm \sqrt{p^{2}-\alpha^{2}}$

To obtain the propagator, first, we need to calculate the integral in Eq. (21) with the

$G_{a d v}(x)=\frac{1}{(2 \pi)^{4}} \int d^{3} p e^{i p r} \int_{a d v} d p_{0} \frac{e^{-i p_{0} x_{0}}}{p^{2}-p_{0}^{2}-\alpha^{2}}$

where Eq. (22) is used for the separation. Then the integration can be evaluated applying the residue theorem. We have two cases. We obtain the advanced propagator if the path of integration runs parallel to the real axis and below both the poles. (In the case of the retarded propagator the path runs above the poles.) Thus, considering the integration $x_{0}>0$ the path is closed on the lower half plane giving null result. In the opposite case, when $x_{0}<0$, there is a non-zero finite contribution of the residues at the poles

$p_{0}= \pm \omega=\sqrt{\boldsymbol{p}^{2}-\alpha^{2}}$ if $\boldsymbol{p}^{2} \geq \alpha^{2}$

and

$p_{0}= \pm i \omega^{\prime}=\sqrt{\boldsymbol{p}^{2}-\alpha^{2}}$ if $\boldsymbol{p}^{2} \leq \alpha^{2}$

After all we can apply the Cauchy's residue theorem for the integration (for the internal integral) with respect to $p_{0}$. We take case if $\boldsymbol{p}^{2} \geq \alpha^{2}$ and $x_{0}<0$. We have two poles (see Eq. (25)), thus the following integral:

$$
\begin{aligned}
& \int_{a d v} d p_{0} \frac{e^{-i p_{0} x_{0}}}{p^{2}-p_{0}^{2}-\alpha^{2}}=2 \pi i \frac{e^{-i \sqrt{\boldsymbol{p}^{2}-\alpha^{2}+i 0} x_{0}}}{-2 \sqrt{\boldsymbol{p}^{2}-\alpha^{2}+i 0}}+2 \pi i \frac{e^{+i \sqrt{\boldsymbol{p}^{2}-\alpha^{2}+i 0} x_{0}}}{2 \sqrt{\boldsymbol{p}^{2}-\alpha^{2}+i 0}} \\
& =-2 \pi \frac{\sin \sqrt{\boldsymbol{p}^{2}-\alpha^{2}+i 0} x_{0}}{\sqrt{\boldsymbol{p}^{2}-\alpha^{2}+i 0}}
\end{aligned}
$$


In the other case, if $\boldsymbol{p}^{2} \leq \alpha^{2}$ and $x_{0}<0$, the calculation is similar with the poles in Eq. (26). It is easy to check that formally the result is the same with the condition $\boldsymbol{p}^{2} \leq \alpha^{2}$. The two cases $\left(\boldsymbol{p}^{2} \geq \alpha^{2}\right.$ and $\left.\boldsymbol{p}^{2} \leq \alpha^{2}\right)$ can be summarized in one expression, i.e., we obtain a 3 rd order integral for the advanced propagator:

$G_{a d v}(x)=-\frac{H\left(-x_{0}\right)}{(2 \pi)^{3}} \int d^{3} p e^{i p r} \frac{\sin \left[\left(\boldsymbol{p}^{2}-\alpha^{2}+i 0\right)^{\frac{1}{2}} x_{0}\right]}{\left(\boldsymbol{p}^{2}-\alpha^{2}+i 0\right)^{\frac{1}{2}}}$

where $H(x)$ is the Heaviside's function which ensures the validity just for the retarded case $x_{0}<0$. Finally, we conclude that this formula is valid for $x_{0}<0$ and for any $\boldsymbol{p}$.

Reversing the previous procedure the retarded propagator $\left(x_{0}>0\right)$ can be also calculated similarly for any $\boldsymbol{p}$

$G_{r e t}(x)=\frac{H\left(x_{0}\right)}{(2 \pi)^{3}} \int d^{3} p e^{i p r} \frac{\sin \left[\left(p^{2}-\alpha^{2}+i 0\right)^{\frac{1}{2}} x_{0}\right]}{\left(p^{2}-\alpha^{2}+i 0\right)^{\frac{1}{2}}}$

Following Feynman's and Wheeler's idea [33, 34], i.e., considering that the propagator is the sum of the half advanced and the half retarded propagator we obtain the propagator

$G(x)=\frac{\operatorname{sgn}\left(x_{0}\right)}{2(2 \pi)^{3}} \int d^{3} p e^{i p r} \frac{\sin \left[\left(\boldsymbol{p}^{2}-\alpha^{2}+i 0\right)^{\frac{1}{2}} x_{0}\right]}{\left(\boldsymbol{p}^{2}-\alpha^{2}+i 0\right)^{\frac{1}{2}}}$

Now, this formula is valid for any $x_{0}$ and for any $\boldsymbol{p}$. Here, the integrals can be rewritten by the Hankel transformation based on Bochner's theorem [35, 36] by which the propagator can be expressed analytically and denoted as

$W^{(4)}(x)=\frac{\alpha}{8 \pi}\left(x_{0}^{2}-r^{2}\right)_{+}^{-\frac{1}{2}} I_{-1}\left(\alpha\left(x_{0}^{2}-r^{2}\right)_{+}^{\frac{1}{2}}\right)$

$I_{-1}(x)$ is the modified Bessel function - taking into account the notations:

$x_{+}^{\beta}=x^{\beta}$ for $x>0$

$x_{+}^{\beta}=0$ for $x<0$

This propagator is often called Wheeler propagator, when the negative sign is in the denominator of the Green function in Eq. (21), i.e., when the denominator is $k^{2}-$ $\alpha^{2}$. As a remark, it is important to emphasize, that the above propagator meets the requirement of causality.

Finally, we can express the resulted electric field $\boldsymbol{E}(\boldsymbol{r}, t)$ generated from an initial charge distribution $\rho\left(x^{\prime}\right)$ during the elapsed time $0 \rightarrow t$ by the application of the calculated propagator:

$\boldsymbol{E}(r, t)=\int-\frac{1}{\varepsilon_{0}} \operatorname{grad} \rho\left(x^{\prime}\right) W^{(4)}\left(x-x^{\prime}\right) d V^{\prime}$ 


\section{Evolution of an Initially Nearly Flat Gaussian Charge Distribution}

On the basis of the previous calculations we can calculate the electric field in view of the initial charge distribution. The question is what kind of process is going within the system governed by the propagator. Now, we imagine a nearly Gaussian charge distribution:

$\rho\left(r^{\prime}, 0\right)=\rho_{0} e^{-a r^{\prime 2}}$

at the initial time 0 in the space coordinate $r_{0}=0$. If the parameter

$a \sim 0$

the charge distribution can be considered practically homogeneous, since we can take that

$$
e^{-a r^{2}} \sim 1
$$

So, if we consider the charge gradient for small values of $a$ we approximate

$\operatorname{grad} \rho\left(r^{\prime}\right)=-2 \rho_{0} a r^{\prime} e^{-a r^{\prime 2}} \sim-2 \rho_{0} a r^{\prime}$

Here, we apply the form of the Wheeler propagator from Eq. (30) for the present analytical calculations. Substituting the calculated charge gradient from Eq. (33) and the Wheeler propagator from Eq. (29) into the expression of $\boldsymbol{E}(r, t)$ in Eq. (31) then we obtain the time evolution of the electric field:

$\boldsymbol{E}(r, t)$

$=\frac{2 \rho_{0} a}{16 \pi^{3} \varepsilon_{0}} \operatorname{Sgn}(t) \int_{V(a l l)} \int_{-\infty}^{\infty} \frac{\sin \left[\left(\boldsymbol{p}^{2}-\alpha^{2}+i 0\right)^{\frac{1}{2}} t\right]}{\left(\boldsymbol{p}^{2}-\alpha^{2}+i 0\right)^{\frac{1}{2}}} e^{i p\left(r-r^{\prime}\right)} \boldsymbol{r}^{\prime} e^{-a r^{\prime 2}} d^{3} p d V^{\prime}$

After the evaluation of the integral and simplifying the mathematical expression the electric field can be analytically expressed as:

$\boldsymbol{E}(r, t)=\frac{\rho_{0} a}{\varepsilon_{0} \alpha} \boldsymbol{r} e^{-a r^{2}} \operatorname{Sgn}(t) \sinh (\alpha|t|)$

It can be read out easily from this exact result that the magnitude of the electric field $\boldsymbol{E}$ follows an exponential behavior. The source of the huge electric field is the enormously growing charge distribution

$\rho(r, t)=\frac{\rho_{0} a}{\alpha}\left(3-2 a r^{2}\right) e^{-a r^{2}} \operatorname{Sgn}(t) \sinh (\alpha|t|)$

which can be obtained by the Maxwell's equation:

$\operatorname{div} \boldsymbol{E}=\frac{\rho}{\varepsilon_{0}}$

It is interesting to see that if we integrate this charge density for the whole space the result is always zero for all positive values of the parameter $a>0$ 
$\int_{0}^{\infty} \rho(r, t) d V=4 \pi \int_{0}^{\infty} \frac{\rho_{0} a}{\alpha}\left(3-2 a r^{2}\right) e^{-a r^{2}} \operatorname{Sgn}(t) \sinh (\alpha|t|) d r=0$

i.e., the conservation law of electric charge is completed, there is only internal movement of the charges - charge disjunction. Applying the continuity relation

$\frac{\partial \rho}{\partial t}+d i v \boldsymbol{J}=0$

and considering equations (3c) and (35) we obtain the current $\boldsymbol{J}(r, t)$

$J(r, t)=-\rho_{0} \operatorname{ar} e^{-a r^{2}} \operatorname{Sgn}(t) \cosh (\alpha|t|)$

Here, we note that calculating the dropped part of Eq. (16)

$-\mu_{0} \frac{\partial J}{\partial t}+\mu_{0} \alpha^{2} \int_{0}^{t} J\left(r, t^{\prime}\right) d t^{\prime}$

with the above solution of the current in Eq. (39), we obtain zero. (This is in line that the current is zero at time 0 .) Thus, we can say that the obtained solution for the electric field in Eq. (35) and for the charge density in Eq. (36) from the cut Klein-Gordon type equation in Eq. (19) can be considered as exact results.

\section{Calculation Result}

The time evolution of the charge density can be also calculated numerically by the propagator form given by Eq. (36). The charge density is homogeneous at the initial time $t_{0}=0$, the process ends in short time $t$. The resulted graphs are shown in Figs. $2-4$ pertaining to time: $0.3,0.7$ and 1.0 . Since the physical situation is spherically symmetric, it is enough to demonstrate the increase of the charge density, as a function of the radius, in different time segments.

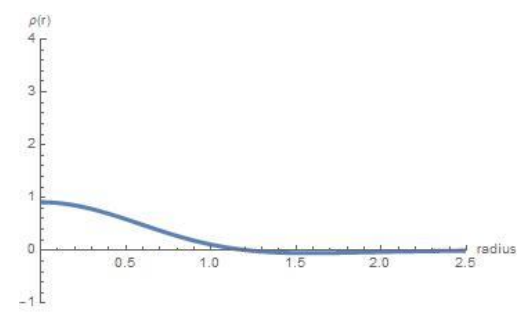

Figure 2

Charge density as a function of the radius $r$ at time $t=0.3$. The charge density and time are considered in natural units. 


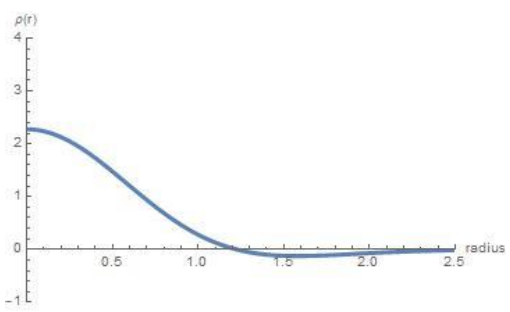

Figure 3

Charge density as a function of the radius $r$ at time $t=0.7$. The charge density and time are considered in natural units.

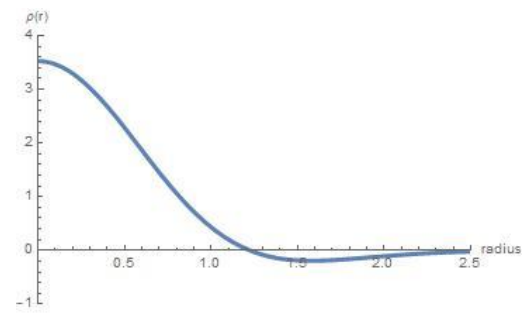

Figure 4

Charge density as a function of the radius $r$ at time $t=1$. The charge density and time are considered in natural units.

This figure demonstrates spectacularly how fast the charge density increases in time. Here, the applied parameters can be taken optionally at the present stage, thus

$\rho_{0}=1$

$a=1$

and

$\alpha=1$

are chosen. This means that the scales are in natural units in the figure. (This a similar assumption when the speed of light is taken $c=1$ or the Planck constant is also $h=1$ in other theories. The tendency does not depend on this choice.) We can see that at the beginning the charge density increases rather slowly comparing the later time, and in a certain time it can grow up in a giant form. During the elapsing time a negative spherically symmetric charge density is collecting with a maximal value at radius $\alpha=1.5$. The process stops at the very short time $\tau$, and it turns back, so finally the system reaches its originally homogeneous charge distribution. It seems from physical reasons natural that the process must be restricted to nano/micro-distances and for short time. 


\section{Conclusions}

As a thought experiment, in the present work, it is shown how a negative mass term Klein-Gordon equation can be deduced in the electrodynamics. This aim could be achieved by adding an appropriate term to one of the Maxwell's equations. It is clear from other experiences of previous studies of mechanical, thermodynamic and field theoretical problems that the appeared term pertains to a repulsive interaction. As a result of the paper, on the one hand, the Wheeler propagator of the process is expressed. We pointed out, on the other hand, that this repulsive force causes a giant charge disjunction on a short range within a short time.

As a final consequence, we can predict that if this short time extreme intensive process (laser light or X-ray, gamma radiation) happens, it can contribute effectively, to the physical behavior of the entire or some small parts of systems, e.g., perhaps on nano/micro-scale, to the electric properties or to other transport phenomena of the few-body systems.

The present calculations do not involve the possibilities of charge oscillations, but this process should remain realistic. This examination and discussion are great challenges for future work.

\section{Acknowledgement}

Support by the Hungarian National Research, Development and Innovation Office of Hungary (NKFIH) Grant Nr. K119442 is acknowledged.

\section{References}

[1] K. Gambár, F. Márkus: A Simple Mechanical Model to Demonstrate a Dynamical Phase Transition, Rep. Math. Phys. 62 (2008) 219

[2] K. Gambár: Change of Dynamics of the Systems: Dissipative - NonDissipative Transition, Informatika 12 (2010) 23

[3] F. Márkus, K. Gambár, Quasiparticles in a Thermal Process, Phys. Rev. E 71 (2005) 066117

[4] K. Gambár, F. Márkus: A Possible Dynamical Phase Transition between the Dissipative and the Non-Dissipative Solutions of a Thermal Process, Phys. Lett. A 361 (2007) 283

[5] F. Márkus, F. Vázquez, K. Gambár: Time Evolution of Thermodynamic Temperature in the Early Stage of Universe, Physica A 388 (2009) 2122

[6] F. Márkus, K. Gambár: Wheeler Propagator of the Lorentz Invariant Thermal Energy Propagation, Int. J. Theor. Phys. 49, (2010) 2065

[7] F. Márkus: “Can a Lorentz Invariant Equation Describe Thermal Energy Propagation Problems?" in Heat Concution - Basic Research (ed. V. S. Vikhrenko), Rijeka: InTech (2011) pp. 155-176 
[8] C. G. Bollini, L. E. Oxman, M. C. Rocca: Coupling of Tachyons to Electromagnetism, Int. J. Theor. Phys. 38 (1999) 777

[9] C. G. Bollini, M. C. Rocca: Wheeler Propagator, Int. J. Theor. Phys. 37 (1998) 2877

[10] C. G. Bollini, J. J. Giambiagi: Dimensional Regularization in Configuration Space, Phys. Rev. D 53 (1996) 5761

[11] C. G. Bollini, M. C. Rocca: Convolution of Lorentz Invariant Ultradistributions and Field Theory, Int. J. Theor. Phys. 43 (2004) 1019

[12] C. G. Bollini, M. C. Rocca: Vacuum State of the Quantum String without Anomalies in any Number of Dimensions, Nuovo Cimento A 110 (1997) 353

[13] C. G. Bollini, M. C. Rocca: Is the Higgs a visible particle? Nuovo Cimento A 110 (1997) 363

[14] C. G. Bollini, L. E. Oxman, M. C. Rocca: Equivalence Theorem for Higher Order Equations, Int. J. Theor. Phys. 37 (1998) 2857

[15] T. Szöllösi, F. Márkus: Searching the laws of Thermodynamics in the Lorentz Invariant Thermal Energy Propagation Equation, Phys. Lett. A 379 (2015) 1960

[16] Ph. M. Morse, H. Feschbach: Methods in Theoretical Physics I McGrawHill, New York, 1953

[17] W. Greiner: Relativistic Quantum Mechanics Berlin, Heidelberg, New York: Springer (2000)

[18] S. R. de Groot, P. Mazur: Non-Equilibrium Thermodynamics North-Holland, Amsterdam, 1962

[19] Sz. Borsányi, A. Patkós, D. Sexty: Phys. Rev. D. 66 (2002) 025014

[20] Sz. Borsányi, A. Patkós, D. Sexty: Phys. Rev. D. 68 (2003) 063512

[21] J. A. Stratton: Electromagnetic Theory New York, London: McGraw-Hill (1941)

[22] J. D. Jackson: Classical Electrodynamics J. Wiley and Sons, New York, 1999

[23] J. Zheng, W. Zhuang, N. Yan, G. Kou, H. Peng, C. McNally, D. Erichsen, A. Cheloha, S. Herek, C. Shi, Y. Shi: Classification of HIV-I-mediated neuronal dendritic and synaptic damage using multiple criteria linear programming, Neuroinformatics 2 (2004) 303

[24] C. Pozna a , R.-E. Precup, J. K. Tar, I. Škrjanc, S. Preitl: New results in modelling derived from Bayesian filtering, Knowledge-Based Systems 23 (2010) 182 
[25] A. Ürmös, Z. Farkas, M. Farkas, T. Sándor, L. T. Kóczy, Á. Nemcsics: Application of self-organizing maps for technological support of droplet epitaxy, Acta Polytechnica Hungarica 14 (2017) 207

[26] S. Vrkalovic, E.-C. Lunca, I.-D. Borlea: Model-free sliding mode and fuzzy controllers for reverse osmosis desalination plants, International Journal of Artificial Intelligence 16 (2018) 208

[27] R. Courant, D. Hilbert: Methods of Mathematical Physics, Vol. II. New York, London: Interscience (1962)

[28] K. Gambár, M. Lendvay, R. Lovassy, J. Bugyjás: Application of Potentials in the Description of Transport Processes, Acta Polytech. Hung. 13 (2016) 173

[29] S. Weinberg: The Quantum Theory of Fields Cambridge: Cambridge Univ. Press. (1995)

[30] M. Srednicki: Quantum field theory Cambridge: Cambridge Univ. Press. (2007)

[31] G. S. Adkins: Phys. Rev. D. 36 (1987) 1929

[32] J. D. Jackson: Am. J. Phys. 70 (2002) 917

[33] J. A. Wheeler, R. P. Feynman: Interaction with the Absorber as the Mechanism of Radiation, Rev. Mod. Phys. 17 (1945) 157

[34] J. A. Wheeler, R. P. Feynman: Classical Electrodynamics in Terms of Direct Interparticle Action, Rev. Mod. Phys. 21 (1949) 425

[35] S. Bochner: Lectures on Fourier Integrals New Jersey: Princeton Univ. Press (1959), pp. 224-230

[36] A. J. Jerri: The Gibbs Phenomenon in Fourier Analysis, splines, and wavelet approximations Dordrecht: Kluwer (1998)

[37] S. Gradshteyn, I. M. Ryzhik: Tables of Integrals, Series, and Products New York: Academic Press (1994) 